\title{
SUPPRESSION OF SPONTANEOUS OSCILLATIONS IN HIGH- FREQUENCY STIMULATED NEURON MODELS
}

\author{
K. Pyragas ${ }^{a}$ and P.A. Tass ${ }^{b, c, d}$ \\ ${ }^{a}$ Center for Physical Sciences and Technology, Sauletekio 3, LT-10222 Vilnius, Lithuania \\ ${ }^{\mathrm{b}}$ Institute of Neuroscience and Medicine - Neuromodulation, Jülich Research Center, Wilhelm-Johnen-Straße, \\ 52425 Jülich, Germany \\ 'Department of Neurosurgery, Stanford University, 450 Serra Mall, Stanford, CA 94305, USA \\ d Department of Neuromodulation, University of Cologne, Albertus-Magnus-Platz, 50923 Cologne, Germany \\ E-mail: pyragas@pfi.lt
}

Received 13 July 2016; accepted 23 September 2016

\begin{abstract}
We analyze the influence of high-frequency current stimulation on spontaneous neuronal activity and show that it may cause a death of spontaneous low-frequency oscillations. We demonstrate the universality of this effect for typical neuron models such as FitzHugh-Nagumo, Morris-Lecar, and Hodgkin-Huxley neurons as well as for the normal form of the supercritical Hopf bifurcation. Using a multiple scale method we separate the solutions of the neuron equations into slow and fast components and derive averaged equations for the slow components. The mechanism of suppression of neuronal activity is explained by an analysis of the bifurcations in the averaged equations governing the dynamics of the slow motion. Our results may contribute to the understanding of therapeutic effects of high-frequency deep brain stimulation, the golden standard for the treatment of medically refractory patients suffering from Parkinson's disease. Furthermore, our study enables hypotheses concerning possible improvements of high-frequency deep brain stimulation.
\end{abstract}

Keywords: neuron models, high-frequency stimulation, Parkinson's disease, nonlinear dynamics, multiple scale method, averaging method

PACS: 05.45.Xt, 87.19.La

\section{Introduction}

The influence of high-frequency excitation on slow dynamical systems is the subject of considerable interest in the field of mechanics for more than one hundred years. The first curious effect was discovered by Stephenson [1, 2], a mathematics lecturer at Manchester University, in 1908. He showed theoretically and experimentally that it is possible to stabilize a rigid pendulum in its inverted, or upside-down, equilibrium position by subjecting the pivot to small vertical oscillations of suitably high frequency. Later on an important contribution to the theory of highfrequency excitation was made by Kapitsa [3, 4h. His approach was based on the concept of direct separation of motions, which implies that the motion under vibration can be presented as a sum of two compo- nents - a fast, "vibrational" component and a "slow" component which changes very little in one period of the vibration. The problems of the modern field of vibrational mechanics [5] include a number of nontrivial vibrational effects such as "flowing up" of a heavy metal ball in a layer of sand, moving of a heavy body or granular material upward along the slope, etc.

In the past two decades great therapeutic progress has been made by applying high-frequency stimulation (HFS) to biological systems [6-8]. To avoid tissue damage one uses biphasic electrical signals with zero mean voltage such that in each period of the high-frequency stimulation the charge induced in the system is balanced. High-frequency $(>100 \mathrm{~Hz})$ deep brain stimulation (DBS) is now recognized as the golden standard for the treatment of patients with medically refractory Parkinson's disease and other movement 
disorders 6-14. Unfortunately, the mechanism of DBS action is still unclear [15-17]. One possible explanation is based on the assumption that the highfrequency stimulation silences the neuronal firing activity of a target area. This hypothesis arose from the observation that the clinical effects induced by lesions and deep brain stimulation of the same target area are similar 10]. Some experiments in vitro [18, 19], in animals [20-22] and in humans [23-26] support the hypothesis of the local inhibition, however, a clear theoretical explanation of this effect is lacking.

The aim of this paper is to apply the ideas of vibrational mechanics to neuronal systems and provide a possible explanation of inhibitory/blocking properties of high-frequency DBS formulated in terms of this field. Note that typical problems of vibrational mechanics are related to passive systems. By contrast, here we deal with active neuronal systems, that spontaneously, i. e. without high-frequency stimulation repetitively generate spikes. We show that the highfrequency stimulation of sufficiently large intensity can silence the repetitive neuronal spiking. The effect is common for different neuron models and can be explained in terms of the stabilization of the neuron resting state, similar to the stabilization of the upsidedown position of a rigid pendulum with a vibrating pivot. In a previous study we have applied a similar vibrational mechanics based approach to investigate the high-frequency stimulation-induced suppression of sustained neuronal spiking in Hodgkin-Huxley and isolated subthalamic nucleus model neurons [27].

The paper is organized as follows. In Section 2 we start our analysis with the generic model of the supercritical Hopf bifurcation extended by a high-frequency excitation term. Here we present the details of the mathematical treatment of the problem based on a two-scale expansion. In the following Sections 3, 4 and 5 we analyze the influence of high-frequency stimulation on the Fitzhugh-Nagumo [28], Morris-Lecar [29] and Hodgkin-Huxley [30] neuron models, respectively. We show that the stabilization of the neuronal resting state may appear by different bifurcation scenarios, depending on a specific neuron model. The paper is finished by conclusions presented in Section 6.

\section{Supercritical Hopf bifurcation}

One of the common mechanisms that gives rise to self-sustained oscillations in nonlinear dynamical systems is the Hopf bifurcation. In order to gain insight into the effect of suppression of low-frequency self-sustained oscillations by HFS we start from a simple model of a forced limit-cycle oscillator that describes the normal form of the supercritical Hopf bifurcation:

$$
\dot{z}=\left[\lambda+\mathrm{i}+(\mathrm{i} \beta-1)|z|^{2}\right] z+a \mathrm{e}^{\mathrm{i} \omega t} .
$$

Here $z$ is a complex variable, $\lambda$ and $\beta$ are real parameters characterizing the Hopf bifurcation. The parameters are normalized in such a way that the Hopf frequency is equal to unity. The last term represents the HFS with the amplitude $a$ and frequency $\omega$. In the free system $(a=0)$, the supercritical Hopf bifurcation appears at $\lambda=0$. For $\lambda<0$ the system has a stable fixed point $z=0$. For $\lambda>0$ it loses the stability and a stable limit cycle $z=r \mathrm{e}^{i \omega 0 t}$ of the radius $r=\sqrt{\lambda}$ and the frequency $\omega_{0}=1+\beta r^{2}$ emerges.

In the presence of HFS, Eq. (1) can be solved approximately by a multiple-scale method [31], or more precisely, by a two-scale expansion. We assume the frequency $\omega$ of the HFS to be a large parameter. In other words, the time $\omega^{-1}$ is much less than all characteristic time-scales of the system. Since the HFS is non-resonant it provides an appreciable effect on the system dynamics only for a sufficiently large amplitude $a$. In the following we suppose that $a$ is proportional to the frequency $\omega, a=A \omega$, where the parameter $A$ is independent of $\omega$. We introduce a fast time $\tau=\omega t$ (the time $t$ is respectively called a slow time) and expand the solution in powers of a small parameter $\omega^{-1}$ :

$$
z(t)=z_{0}(t, \tau)+\omega^{-1} z_{1}(t, \tau) \ldots .
$$

We suppose that the functions $z_{0}, z_{1}, \ldots$ are $2 \pi$ periodic in $\tau: z_{0}(t, \tau+2 \pi)=z_{0}(t, \tau), z_{1}(t, \tau+2 \pi)=z_{1}(t, \tau), \ldots$. This means that the solution is periodic on the fast time scale with the period equal to the period of HFS. We treat $t$ and $\tau$ as if they were independent variables and obtain

$$
\dot{z}=\omega \frac{\partial z_{0}}{\partial \tau}+\frac{\partial z_{0}}{\partial t}+\frac{\partial z_{1}}{\partial \tau} \cdots .
$$

Substituting (2) into (1) using (3) and equating terms proportional to $\omega^{1}$ and $\omega^{0}$ (recall that $a=A \omega$ ) gives

$$
\begin{aligned}
& \frac{\partial z_{0}}{\partial \tau}=A \mathrm{e}^{\mathrm{i} \tau}, \\
& \frac{\partial z_{0}}{\partial t}+\frac{\partial z_{1}}{\partial \tau}=\left[\lambda+(\alpha+\mathrm{i} \beta)\left|z_{0}\right|^{2}\right] z_{0} .
\end{aligned}
$$

The solution of Eq. (4) is

$$
z_{0}(t, \tau)=\bar{z}(t)-\mathrm{i} A \mathrm{e}^{\mathrm{i} \tau},
$$

where $\bar{z}(t)$ is any function of the slow time $t$. To derive an equation for this function we substitute (6) into (5), 


$$
\frac{\mathrm{d} \bar{z}}{\mathrm{~d} t}+\frac{\partial z_{1}}{\partial \tau}=\left[\lambda+\mathrm{i}+(\mathrm{i} \beta-1)\left|\bar{z}-\mathrm{i} A \mathrm{e}^{\mathrm{i} \tau}\right|^{2}\right]\left(z_{0}-\mathrm{i} A \mathrm{e}^{\mathrm{i} \tau}\right),
$$

and average it over the period of fast stimulation, i. e. multiply it by $1 / 2 \pi$ and integrate over the period of fast time $\int_{0}^{2 \pi} \mathrm{d} \tau \cdots$ :

$$
\dot{\bar{z}}=\left[\lambda-2 A^{2}+\mathrm{i}\left(1+2 A^{2} \beta\right)+(\beta-1)|\bar{z}|^{2}\right] \bar{z} \text {. }
$$

Finally, an approximate solution of Eq. (1) can be presented as a sum of the slow and fast components (this result is in agreement with the Kapitsa's concept and is typical for the problems of vibrational mechanics [5]):

$$
z(t) \approx \bar{z}(t)-\mathrm{i} A \mathrm{e}^{\mathrm{i} \omega t} .
$$

The fast "vibrational" component is $-\mathrm{i} A \mathrm{e}^{\mathrm{i} \omega t}$ and the slow component $\bar{z}(t)$ satisfies the averaged equation (8). The relationship between initial conditions of Eqs. (8) and (1) is $\bar{z}(0)=z(0)+\mathrm{i} A$. Note that Eq. (8) is autonomous - contrary to Eq. (1), it does not contain the HFS term $a \mathrm{e}^{\mathrm{i} \omega t}$. The solution $\bar{z}(t)$ of the averaged equation (8) changes only slightly in one period of HFS; it approximates the moving average of the solution $z(t)$ of the original equation (1):

$$
\bar{z}(t) \approx \int_{t-T / 2}^{t+T / 2} z\left(t^{\prime}\right) \mathrm{d} t^{\prime}
$$

The averaged equation (8) is equivalent to the equation of the free oscillator that describes the normal form of Hopf bifurcation. However, its parameters are modified by additional terms proportional to $A^{2}=(a / \omega)^{2}$. These terms are related to HFS. In vibrational mechanics, such terms are referred to as the "vibrational forces". The supercritical Hopf bifurcation in system (8) is shifted in comparison to the free system. It appears for $\lambda=2 A^{2}$ and its Hopf frequency is $1+2 A^{2} \beta$. For $\lambda=2 A^{2}$ the slow motion converges to the stable fixed point $\bar{z}=0$, while for $\lambda>2 A^{2}$ the fixed point is unstable and we have the periodic slow motion around the stable limit cycle $\bar{z}=\bar{r} \mathrm{e}^{\mathrm{i}-\omega 0 t}$ of radius $\bar{r}=\sqrt{\lambda-2 A^{2}}$ and frequency $\bar{\omega}_{0}=1+2 A^{2} \beta+\beta \bar{r}^{2}=1+\beta \lambda$. The frequency of the limit cycle is independent of HFS.

In Fig. 1(a) we see that the increase of the intensity $A=a / \omega$ of HFS causes a reverse supercritical Hopf bifurcation in the slow dynamics of the forced oscillator. For $A>\sqrt{\lambda / 2}$ the radius $\bar{r}$ of the limit cycle vanishes and the fixed point $\bar{z}=0$ becomes stable. In Fig. 1(b) we demonstrate the death of slow limit cycle oscillations after switching on the HFS. The averaged equation (8) describes well the transient dynamics towards the death of slow oscillations.
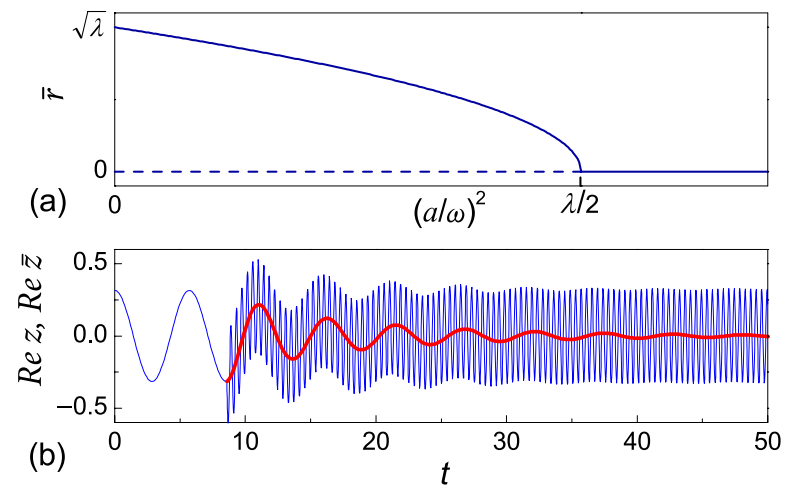

Fig. 1. (a) Bifurcation diagram of the averaged equation (8). With increasing intensity of the HFS the radius of the limit cycle decreases according to $\bar{r}=\sqrt{\lambda-2(a / \omega)^{2}}$. The dashed (solid) horizontal line represents the unstable (stable) fixed point $\bar{z}=0$. (b) The death of low-frequency oscillations in system (1) for $\lambda=0.1, \beta=1$ and $\omega=15$. For $t<t_{s}=8.6$ the free system $(a=0)$ moves along the stable limit cycle. For $t>t_{s}$ HFS with amplitude $a=4.5$ is switched on. The thin (blue online) line shows the solution of Eq. (1). The bold (red) line shows the solution of the averaged equation (8) for $t>t_{s}$. (All quantities are in dimensionless units.)

This example shows that the dependence of bifurcation parameters on "vibrational forces" allows us to control the slow dynamics of the system via HFS and even induce qualitative changes in this dynamics. The total dynamics consists of a sum of the slow and fast motions. The suppression of the slow motion as opposed to the fast motion will be discussed from a neuroscientific standpoint below.

\section{FitzHugh-Nagumo model}

One of the simplest neuron models is described by the FitzHugh-Nagumo equations [28]. These equations conceptually isolate the essential mathematical properties of excitation and propagation from the electrochemical properties of the sodium and potassium ion flow. In the presence of HFS $a \cos (\omega t)$ the equations read

$$
\begin{aligned}
& \dot{v}=v-v^{3} / 3-w+I+a \cos (\omega t), \\
& \dot{w}=\varepsilon(b+v-c w) .
\end{aligned}
$$

Here $v$ is a membrane potential and $w$ stands for a recovery variable. We take the following values of the parameters: $b=0.7, c=0.8, \varepsilon=0.08$. The behaviour of the free system $(a=0)$ depends on the DC current $I$. For sufficiently large values of this current the system generates periodical spikes. The phase portrait of the free system representing such a behaviour is 
shown in Fig. 2(a). The nulclines $\left(\dot{v}=0: w=v-v^{3} / 3+I\right.$ and $\dot{w}=0: w=(v+b) / c)$ of the system intersect on the increasing part of the $\mathrm{N}$-shaped $v$-nulcline. As a result, the system has an unstable fixed point and a stable limit cycle. Due to the smallness of the parameter $\varepsilon$ the phase point moves along the limit cycle nonmonotonously and the system experiences relaxation oscillations.

Our aim is to show that HFS may suppress the spontaneous oscillations of the system and explain the mechanism of this suppression. In the Appendix we present the derivation of averaged equations for a general neuron model. Applying these general results to Eqs. (11) we can write an approximate solution in the form

$$
\begin{aligned}
& v(t) \approx \bar{v}(t)+A \sin (\omega t), \\
& w(t) \approx \bar{w}(t),
\end{aligned}
$$

where $A=a / \omega$ and the variables $\bar{v}$ and $\bar{w}$ satisfy the averaged equations (A.8). To write these equations in the explicit form we note that here the functions $f$ and $g$ are $f(v, w)=v-v^{3} / 3-w+I$ and $g(v, w)=\varepsilon(b+v-c w)$. Averaging of these functions over the fast time can be performed analytically: $\langle f(\bar{v}+A \sin \tau, \bar{w})\rangle_{\tau}=\bar{v}\left(1-A^{2} / 2\right)-\bar{w}^{3} / 3 \bar{w}+I$ and $\langle g(\bar{v}+A \sin \tau, \bar{w})\rangle_{\tau}=\varepsilon(b+\bar{v}-c \bar{w})$. Then the averaged equations take the form

$$
\begin{aligned}
& \dot{\bar{v}}=\bar{v}\left(1-A^{2} / 2\right)-\bar{v}^{3} / 3-\bar{w}+I, \\
& \dot{\bar{w}}=\varepsilon(b+\bar{v}-c \bar{w}) .
\end{aligned}
$$

For $a=0$ the averaged equations (13) are equivalent to the original system (3). Thus without HFS the phase portraits of systems (11) and (13) coincide (Fig. 2(a)). With the increase of $a$ the linear nulcline $\bar{w}=(\bar{v}+b) / c$ remains unchanged while the $\mathrm{n}$-shaped nulcline $\bar{w}=\bar{v}\left(1-\mathrm{A}^{2} / 2\right) \bar{v}^{3} / 3+I$ varies due to the "vibrational force" $A^{2} / 2$. As a result, the limit cycle in the phase space of averaged equations shrinks and engulfs the fixed point rendering it stable (Fig. 2(b, c)). After this, the stable fixed point is the only stable attractor of the averaged system. Thus the increase of HFS intensity leads to the reverse supercritical Hopf bifurcation similar to that described in the previous section. This scenario explains the mechanism of suppression of the low-frequency oscillations in the highfrequency stimulated Fitzhugh-Nagumo neuron.

The transient dynamics of the system (11) towards the death of spontaneous low-frequency oscillations after switching on the HFS is shown in Fig. 3. The averaged equations (13) describe the system dynamics well even in the case of relaxation oscillations. Although the free system has two different time scales (the slow $w$ variable and the fast $v$ variable), the averaged equations are correct when $\omega^{-1}$ is less than the characteristic time scale of the fast variable.

In Fig. 3 we see that HFS induces a relatively large high-frequency component in the membrane potential $v$, while for the recovery variable $w$ this component is small. This is in agreement with the approximate solution (12). The reason is that HFS is applied only to Eq. (11a) of the membrane potential and does not directly influence the recovery variable $w$.
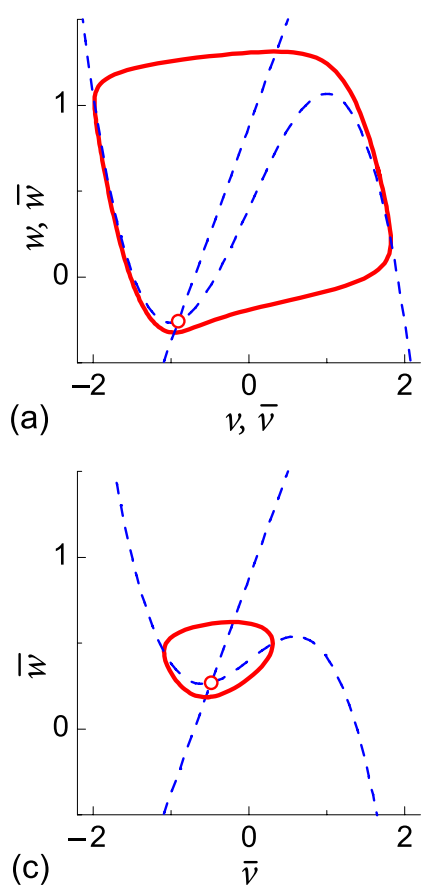

(b)
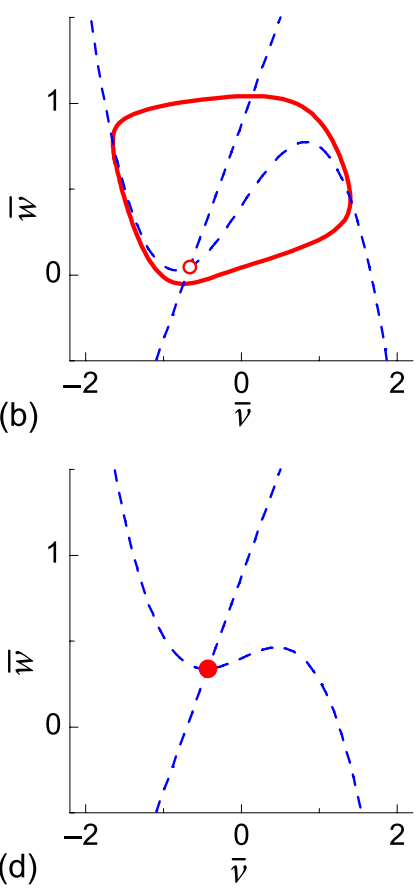

Fig. 2. Phase portraits of the averaged equations (13) of the FitzHugh-Nagumo neuron for $b=0.7$, $a=0.8, \varepsilon=0.08, I=0.4$ and $\omega=5$. The nulclines are shown by dashed (blue online) lines, the limit cycles are depicted by solid (red) curves, the stable and unstable fixed points (the intersection points of the nulclines) are presented by solid (red) dots and open circles, respectively. (a) $a=0$ (this phase portrait coincides with that of system (11)); (b) $a=4$; (c) $a=5.7$; (d) $a=6.3$. (All quantities are in dimensionless units.) 

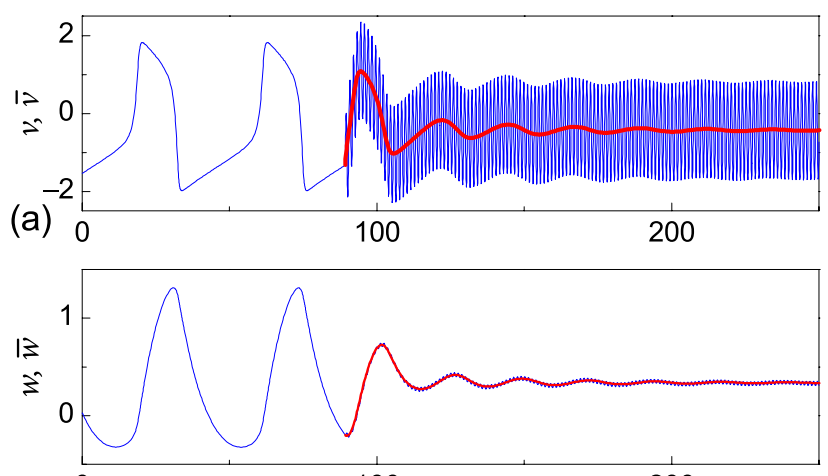

(b)

$t$

Fig. 3. (a) The death of spontaneous low-frequency oscillations under HFS in the FitzHugh-Nagumo system. For $t<t_{s}=90$ the free system $(a=0)$ moves along the stable limit cycle shown in Fig. 2(a). For $t>t_{s}$ the HFS of amplitude $a=6.3$ is switched on. Other parameters are the same as in Fig. 2. The thin (blue) lines show the solution of Eqs. (11). The bold (red) lines show the solution of the averaged equations (2) for $t>t_{s}$. The "vibrational" component for the $w$ variable is so small that the oscillations are covered by the bold red line of the averaged variable $\bar{w}$. (All quantities are in dimensionless units.)

\section{Morris-Lecar model}

A more realistic neuron model than that described in the previous section is given by the Morris-Lecar equations [29]. Historically, these equations were derived from an experimental study of the excitability of the giant muscle fiber of the huge Pacific barnacle. In fact, these equations represent a two-dimensional reduction of the four-dimensional Hodgkin-Huxley neuron model that will be considered in the next section. In the presence of HFS the Morris-Lecar equations are

$$
\begin{aligned}
& C \dot{v}=-g_{\mathrm{Ca}} m_{\infty}(v)\left(v-v_{\mathrm{Ca}}\right)-g_{\mathrm{K}} w\left(v-v_{\mathrm{K}}\right) \\
& -g_{\mathrm{L}}\left(v-v_{\mathrm{L}}\right)+I+a \cos (\omega t), \\
& \dot{w}=\phi\left[w_{\infty}(v)-w\right] / \tau_{w}(v) .
\end{aligned}
$$

Here $C$ is the membrane capacitance. The parameters $v_{\mathrm{Ca}}, v_{\mathrm{K}}$ and $v_{\mathrm{L}}$ are the equilibrium potentials of $\mathrm{Ca}^{2+}$, $\mathrm{K}^{+}$and leak currents, respectively; $g_{\mathrm{Ca}}, g_{\mathrm{K}}$ and $g_{\mathrm{L}}$ are the maximum conductance of the corresponding ionic currents. The parameter $\phi$ defines the characteristic time scale of the recovery variable. The voltagedependent functions are

$$
m_{\infty}(v)=\frac{1}{2}\left[1+\tanh \left(\frac{v-v_{1}}{v_{2}}\right)\right],
$$

$$
\begin{aligned}
& w_{\infty}(v)=\frac{1}{2}\left[1+\tanh \left(\frac{v-v_{3}}{v_{4}}\right)\right], \\
& \tau_{w}(v)=\left[\cosh \left(\frac{v-v_{3}}{2 v_{4}}\right)\right]^{-1} .
\end{aligned}
$$

For a sufficiently large frequency $\omega$ an approximate solution of Eqs. (14) is

$$
\begin{aligned}
& v(t) \approx \bar{v}(t)+A \sin (\omega t), \\
& w(t) \approx \bar{w}(t),
\end{aligned}
$$

where $A=a / \omega C$ and the variables $\bar{v}$ and $\bar{w}$ satisfy the averaged equations:

$$
\begin{aligned}
& \dot{\bar{v}}=g_{\mathrm{Ca}} \bar{\mu}(\bar{v}, A)+g_{\mathrm{K}} \bar{w}\left(\bar{v}-v_{\mathrm{K}}\right) \\
& -g_{L}\left(\bar{v}-v_{\mathrm{L}}\right)+I, \\
& \dot{\bar{w}}=\bar{\alpha}(\bar{v}, A)-\bar{\beta}(\bar{v}, A) \bar{w} .
\end{aligned}
$$

Here we have introduced the functions

$$
\begin{aligned}
& \bar{\mu}(v, A)=\langle\mu(\bar{v}+A \sin \tau)\rangle_{\tau^{\prime}} \\
& \bar{\alpha}(v, A)=\langle\alpha(\bar{v}+A \sin \tau)\rangle_{\tau} \\
& \bar{\beta}(v, A)=\langle\beta(\bar{v}+A \sin \tau)\rangle_{\tau}
\end{aligned}
$$

where

$$
\begin{aligned}
& \mu(v)=m_{\infty}(v)\left(v-v_{\mathrm{Ca}}\right), \\
& \alpha(v)=\phi w_{\infty}(v) / \tau_{\infty}(v), \\
& \beta(v)=\phi / \tau_{\infty}(v) .
\end{aligned}
$$

Unfortunately, we cannot derive explicit analytical expressions for the averaged functions (18) in the general case. An approximate analytical estimation of these functions can be obtained by means of a Taylor series expansion only for small values of $A$. However, the effect of suppression of spontaneous oscillations appears for rather large amplitudes $A$ when the Taylor series approximation fails. Therefore, the integration of the averaged equations (17) requires a numerical computation of the averaged functions (18) inside the Runge-Kutta algorithm.

Depending on the specific values of the parameters the free $(a=0)$ Morris-Lecar model may demonstrate the behaviour typical of the so-called type I or type II neurons [32]. Such a classification 
is based on the difference of the onset of autonomous repetitive firing. By increasing the DC current $I$ the neuron with type I excitability starts to fire repetitively with an almost zero frequency. According to the bifurcation theory, the firing results from a saddle-node bifurcation on an invariant circle. In contrast, the type II neuron starts to fire with a finite frequency. Here the onset of firing can be caused by a Hopf bifurcation.

We have analyzed the effect of HFS for both types of excitability and in both cases we observed the effect of suppression of the repetitive firing. Below we present the results for the type I excitability. An evolution of the phase portrait of the averaged system (17) is shown in Fig. 4 for the fixed value of the DC current $I$ and the varying intensity of the HFS current. The averaged dynamics of the Morris-Lecar neuron experiences a reverse supercritical Hopf bifurcation similar to the Fitzhugh-Nagumo neuron. Thus, the mechanism of suppression of the spontaneous firing is identical for these two different neuron models.

The transient dynamics of the Morris-Lecar neuron towards the death of spontaneous firing shown in Fig. 5 is also similar to that observed in the Fitzhugh-Nagumo neuron (cf. Fig. 3). Again the solution of averaged Eqs. (17) fits well to the averaged dynamics of original Eqs. (14).
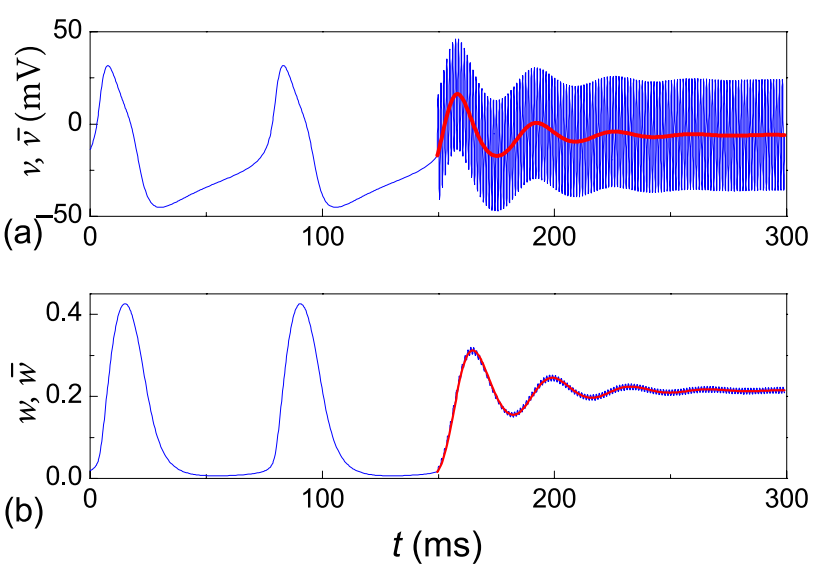

Fig. 5. (a) The death of spontaneous firing under HFS in the Morris-Lecar neuron. For $t<t_{s}=150 \mathrm{~ms}$ the free system $(a=0)$ generates spontaneous spikes corresponding to the stable limit cycle shown in Fig. 4(a). For $t>t$ s the HFS of amplitude $a=3 \mathrm{~mA} / \mathrm{cm}^{2}$ is switched on. Other parameters are the same as in Fig. 4. Thin (blue) and bold (red) lines show the solutions of Eqs. (14) and (17), respectively.

\section{Hodgkin-Huxley model}

As a last example, we consider the Hodgkin and Huxley [30] model. The model was originally developed for the squid giant axon. In the presence of HFS the model reads:

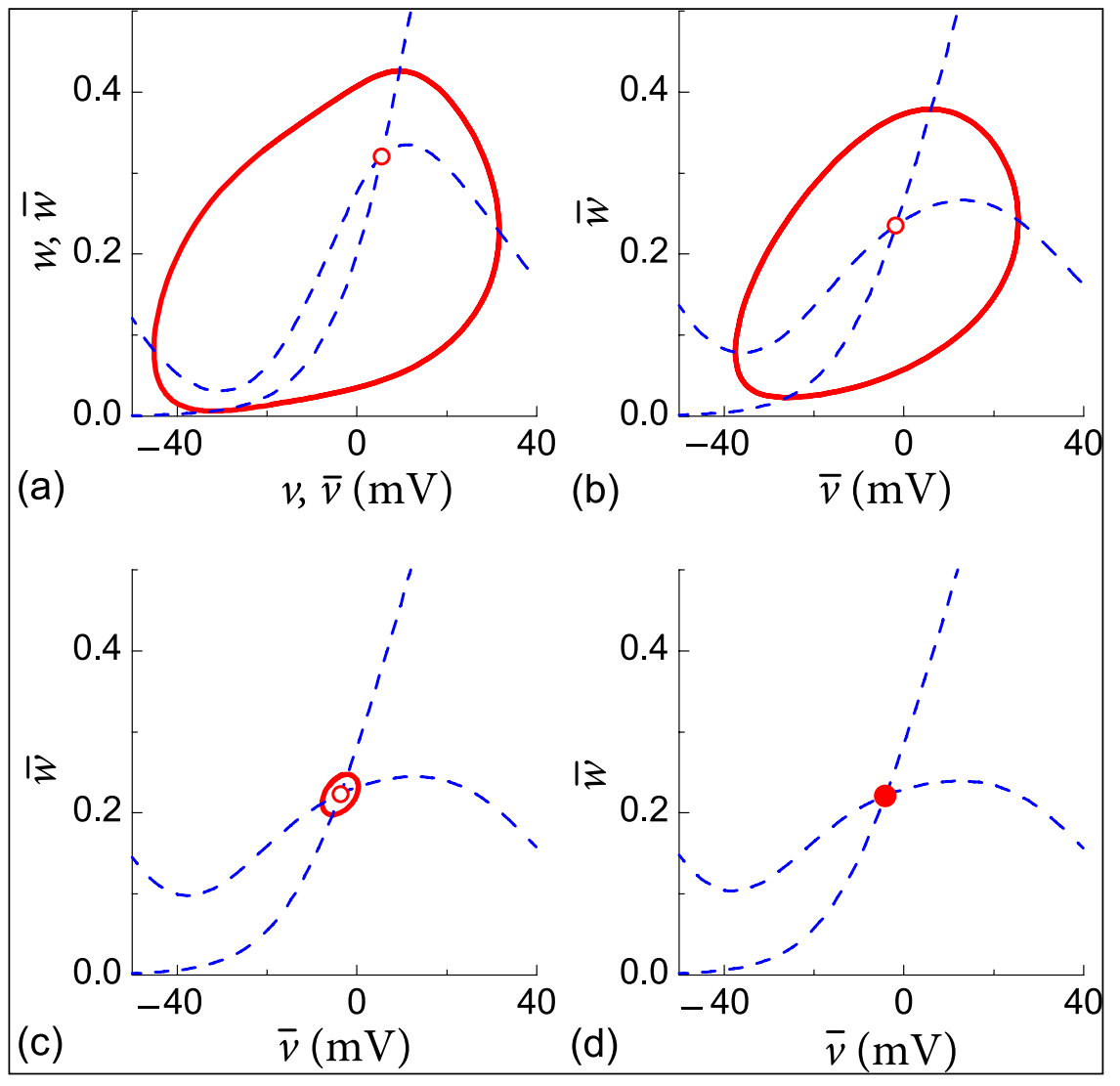

Fig. 4. The same as in Fig. 2 but for the averaged equations (17) of the Morris-Lecar neuron. The set of the parameters correspond to the type I excitability: $g_{\mathrm{Ca}}=44 \mathrm{mS} / \mathrm{cm}^{2}, g_{\mathrm{K}}=8 \mathrm{mS} /$ $\mathrm{cm}^{2}, g_{\mathrm{L}}=2 \mathrm{mS} / \mathrm{cm}^{2}, v_{\mathrm{Ca}}=120 \mathrm{mV}$, $v_{\mathrm{K}}=-80 \mathrm{mV}, v_{\mathrm{L}}=-60 \mathrm{mV}$, $v_{1}=-2 \mathrm{mV}, v_{2}=18 \mathrm{mV}, v_{3}=12 \mathrm{mV}$, $v_{4}=17.4 \mathrm{mV}, \phi=0.0667 \mathrm{~ms}^{-1}$, $C=20 \mu \mathrm{F} / \mathrm{cm}^{2}$. The DC current is $I=50 \mu \mathrm{A} / \mathrm{cm}^{2}$. The frequency of HFS is $\omega=5 \mathrm{kHz}$ and the amplitude is the following: (a) $a=0$ (this phase portrait coincides with that of the system (14)); (b) $a=2 \mathrm{~mA} / \mathrm{cm}^{2}$; (c) $a=2.4 \mathrm{~mA} /$ $\mathrm{cm}^{2}$; (d) $a=2.5 \mathrm{~mA} / \mathrm{cm}^{2}$. 


$$
\begin{aligned}
& C \dot{v}=-g_{\mathrm{Na}} m^{3} h\left(v-v_{\mathrm{Na}}\right)-g_{\mathrm{K}} n^{4}\left(v-v_{\mathrm{K}}\right) \\
& -g_{\mathrm{L}}\left(v-v_{\mathrm{L}}\right)+I+a \cos (\omega t), \\
& \dot{m}+\alpha_{m}(v)(1-m)-\beta_{m}(v) m, \\
& \dot{h}+\alpha_{h}(v)(1-h)-\beta_{h}(v) h, \\
& \dot{n}+\alpha_{n}(v)(1-n)-\beta_{n}(v) n .
\end{aligned}
$$

Here $C=1 \mu \mathrm{F} / \mathrm{cm}^{2}$ is the membrane capacitance and $v$ is the membrane potential measured in $\mathrm{mV}$. The parameters $v_{\mathrm{Na}}=115 \mathrm{mV}, v_{\mathrm{K}}=-12 \mathrm{mV}$ and $v_{\mathrm{L}}=10.6 \mathrm{mV}$ are the equilibrium potentials of $\mathrm{Na}^{+}, \mathrm{K}^{+}$, and leak currents, respectively. $g_{\mathrm{Na}}=120 \mathrm{mS} / \mathrm{cm}^{2}, g_{\mathrm{K}}=36 \mathrm{mS} / \mathrm{cm}^{2}$ and $g_{\mathrm{L}}=0.3 \mathrm{mS} / \mathrm{cm}^{2}$ are the maximum conductance of the corresponding ionic currents. $0 \leq m \leq 1$ and $0 \leq h \leq 1$ are the gating variables responsible, respectively, for activation and inactivation of the $\mathrm{Na}^{+}$current, and $0 \leq n \leq 1$ is the gating variable representing activation of the $\mathrm{K}^{+}$current. The rate parameters $\alpha_{x}, \beta_{x}(x=m, h, n)$ measured in $\mathrm{ms}^{-1}$ are the following functions of the membrane potential:

$$
\begin{aligned}
& \alpha_{m}(v)=(2.5-0.1 v) /[\exp (2.5-0.1 v)-1] \\
& \beta_{m}(v)=4 \exp (-v / 18) \\
& \alpha_{h}(v)=0.07 \exp (-v / 20) \\
& \beta_{h}(v)=1 /[\exp (3-0.1 v)+1] \\
& \alpha_{n}(v)=(0.1-0.01 v) /[\exp (1-0.1 v)-1] \\
& \beta_{n}(v)=0.125 \exp (-v / 80)
\end{aligned}
$$

Here the voltage scale is shifted in such a way that the membrane resting potential without external currents $(I=a=0)$ is zero.

In contrast to the previous neuron models, the Hodgkin-Huxley neuron (20) is described by four dynamical variables. In order to apply the general theory presented in the Appendix, we note that the last three equations $(20 \mathrm{~b}-\mathrm{d})$ can be interpreted as a one vector-equation for the three-dimensional recovery variable $w=(m, h, n)$. Then the approximate solution of Eqs. (20) can be written as

$$
\begin{aligned}
& v(t) \approx \bar{v}(t)+A \sin (\omega t), \\
& m(t) \approx \bar{m}(t), \\
& h(t) \approx \bar{h}(t),
\end{aligned}
$$

$$
n(t) \approx \bar{n}(t)
$$

where $A=a / \omega C$ and the slow variables $\bar{v}, \bar{m}, \bar{h}$ and $\bar{n}$ satisfy the averaged equations:

$$
\begin{aligned}
& \dot{\bar{v}}=-g_{\mathrm{Na}} \bar{m}^{3} \bar{h}\left(\bar{v}-v_{\mathrm{Na}}\right)-g_{\mathrm{K}} \bar{n}^{4}\left(\bar{v}-v_{\mathrm{K}}\right) \\
& -g_{\mathrm{L}}\left(-v-v_{\mathrm{L}}\right)+I, \\
& \dot{\bar{m}}=\bar{\alpha}_{m}(\bar{v}, A)(1-\bar{m})-\bar{\beta}_{m}(\bar{v}, A) \bar{m}, \\
& \dot{\bar{h}}=\bar{\alpha}_{h}(\bar{v}, A)(1-\bar{h})-\bar{\beta}_{h}(\bar{v}, A) \bar{h}, \\
& \dot{\bar{n}}=\bar{\alpha}_{n}(\bar{v}, A)(1-\bar{n})-\bar{\beta}_{n}(\bar{v}, A) \bar{n} .
\end{aligned}
$$

These equations are equivalent to the original Hodgkin-Huxley equations (21), but there is no high-frequency stimulation current and the original rate coefficients $(21)$ are replaced by the averaged coefficients $\bar{\alpha}_{x}, \bar{\beta}_{x}(x=m, h, n)$ :

$$
\begin{aligned}
& \bar{\alpha}_{x}(\bar{v}, A)=\left\langle\alpha_{x}(\bar{v}+A \sin \tau)\right\rangle_{\tau}, \\
& \bar{\beta}_{x}(\bar{v}, A)=\left\langle\beta_{x}(\bar{v}+A \sin \tau)\right\rangle_{\tau} .
\end{aligned}
$$

If the stimulation amplitude is not very large, the averaged rate coefficients can be estimated analytically by means of the Taylor series expansion:

$$
\begin{aligned}
& \bar{\alpha}_{x}(\bar{v}, A) \approx \alpha_{x}(\bar{v})+A^{2} \alpha_{x}^{\prime \prime}(\bar{v}) / 4, \\
& \bar{\beta}_{x}(\bar{v}, A) \approx \beta_{x}(\bar{v})+A^{2} \beta_{x}^{\prime \prime}(\bar{v}) / 4 .
\end{aligned}
$$

Here $\alpha_{x}^{\prime \prime}$ and $\beta_{x}^{\prime \prime}$ are the second derivatives of the corresponding functions.

In contrast to the Morris-Lecar model, here the death of the spontaneous firing occurs at relatively small values of the amplitude $A$, so that the approximations (25) give the adequate description for all the effects considered below. Note that all functions in Eqs. (23), (25) and (21), that comprise a model for the slow averaged dynamics of the Hodgkin-Huxley neuron, are defined analytically.

For the given parameter values the HodgkinHuxley neuron demonstrates the type II excitability. When increasing the DC current $I$, the neuron starts repetitive firing due to the subcritical Hopf bifurcation. The bifurcation appears for $I \approx 9.8 \mu \mathrm{A} / \mathrm{cm}^{2}$. Figure 6 (a) shows the two-dimensional projection $(v, n)$ of the phase portrait of free $(a=0)$ system (20) for $I=20 \mu \mathrm{A} / \mathrm{cm}^{2}$, i. e. above the threshold of the onset of spontaneous firing. Without HFS $(a=0)$ the same result is obtained from the averaged equations (23). The phase portrait is characterized by the unstable 

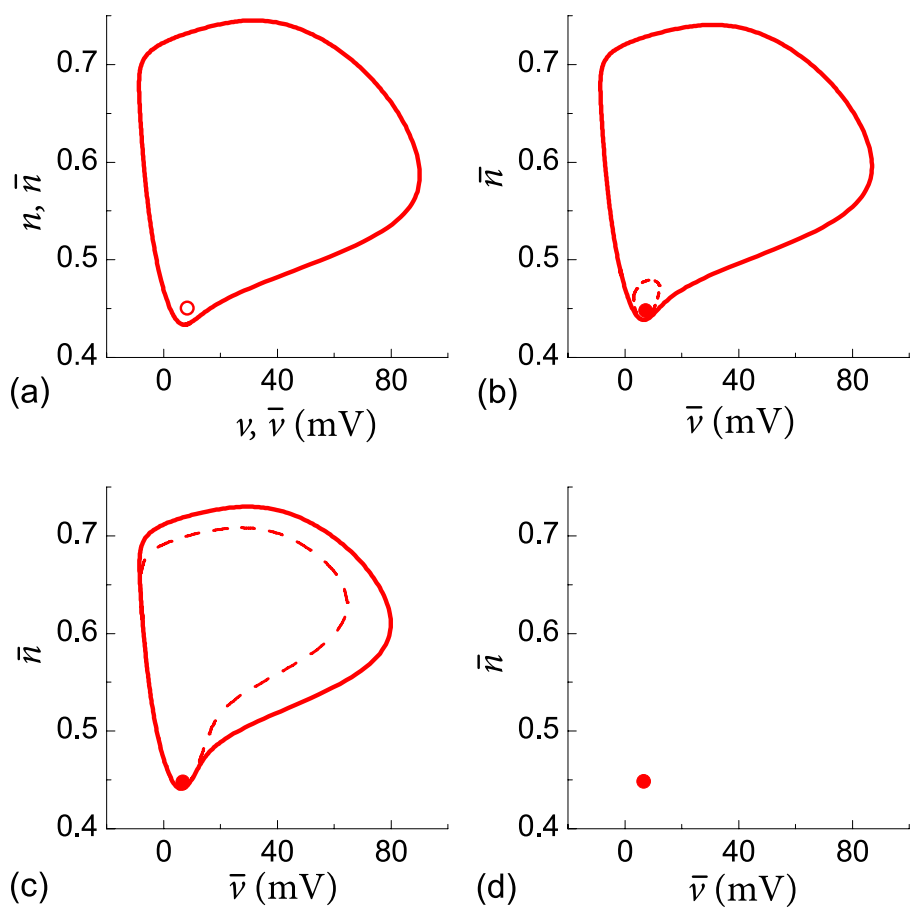

Fig. 6. The $(\bar{v}, \bar{n})$ projections of the averaged equations (23) of the Hodgkin-Huxley neuron for different intensities of HFS: (a) $A=0$ (this phase portrait coincides with that of the system (20)); (b) $A=12 \mathrm{mV}$ (just after the subcritical Hopf bifurcation); (c) $A=15 \mathrm{mV}$ (just before the double-cycle bifurcation); (d) $A=16 \mathrm{mV}$ (just after the double-cycle bifurcation). The parameters of the Hodgkin-Huxley model are given in the text.

resting state and the stable limit cycle responsible for the neuron repetitive firing.

The subsequent Figs. 6(b-d) show the evolution of the phase portrait of averaged Eqs. (5) emerging with increasing HFS intensity. Here the death of spontaneous oscillations is characterized by a more complicated scenario as in the models considered in the previous sections. Now the fixed point becomes stable through a reverse subcritical Hopf bifurcation that appears for $A=A_{\text {subH }} \approx 11.16 \mathrm{mV}$. This bifurcation gives birth to a small unstable limit cycle, which expands with a further increase of HFS intensity. For $A=A_{\mathrm{dc}} \approx 15.17 \mathrm{mV}$ the stable and unstable limit cycles collide and disappear trough a double-cycle bifurcation. After this bifurcation, the stable fixed point is the only stable attractor of the averaged system.

Summarizing the described bifurcations, the behaviour of the Hodgkin-Huxley neuron under HFS is as follows. For small intensities $A<A_{\text {subH }}$ the neuron remains in the regime of spontaneous firing. For large intensities $A>A_{\mathrm{dc}}$ the neuron definitely stops firing and settles at a stable resting state. The moderate intensities $A_{\text {subH }}<A<A_{\mathrm{dc}}$ lead to a bistable regime-depending on the initial conditions the neuron may approach either the stable resting state or the stable limit cycle of spontaneous firing.

In Fig. 7 we show how the dynamics of the Hodgkin-Huxley neuron changes when HFS is switched on at moderate intensities $A=11.2 \mathrm{mV}$, which correspond to the bistable regime. Although the resting state is stable for this value of $A$, the neuron remains firing after switching on the HFS. This result is independent of the moment of switching on the HFS. To explain this result we note that in any moment of the switching on the HFS the initial conditions are placed on the unperturbed $(A=0)$ stable limit cycle. Since the stable limit cycle changes only slightly with an increase of $A$ (compare Figs. $6(\mathrm{a}-\mathrm{c})$ ), the nearest stable state for the perturbed system is the perturbed limit cycle.

The suppression of spontaneous firing can always be achieved by using HFS of a sufficiently large intensity. Above the threshold of the double-cycle bifurcation $A>A_{\mathrm{dc}}$ the system reaches the only stable steady-state independently of the initial conditions. In Fig. 8 we demonstrate that the stable resting state can be reached even for moderate intensities $A_{\text {subH }}<A<A_{\mathrm{dc}}$. The problem related to the bistability is avoided by using a simple two-step algorithm. In the first step, we switch on the HFS at a large amplitude $A>A_{\mathrm{dc}}$ and reach the stable resting state. In the second step, we decrease the HFS amplitude to moderate values $A_{\text {subH }}<A<A_{\mathrm{dc}}$ where this state is still stable. As a result, we can suppress the spontaneous firing and maintain the stable resting state using the smallest permissable amplitude, slightly above the threshold of the subcritical Hopf bifurcation.

\section{Conclusions}

We have shown that the neuronal spontaneous firing can effectively be suppressed by a charge-balanced high-frequency stimulation current. The universality of this effect is demonstrated by different neuron models, namely, the two-dimensional FitzHugh-Na- 

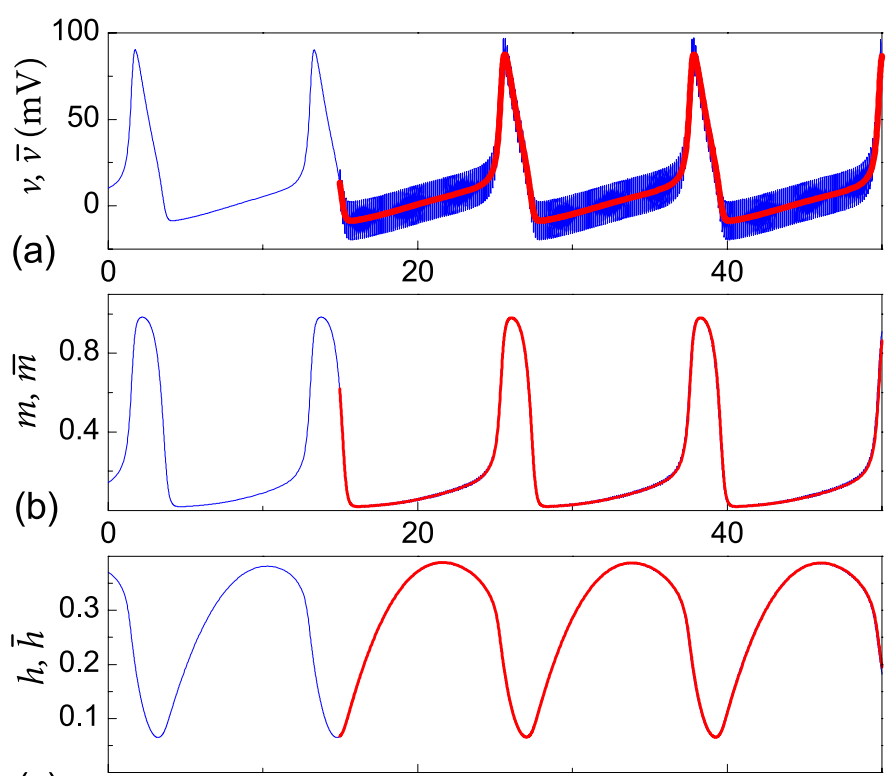

(c)
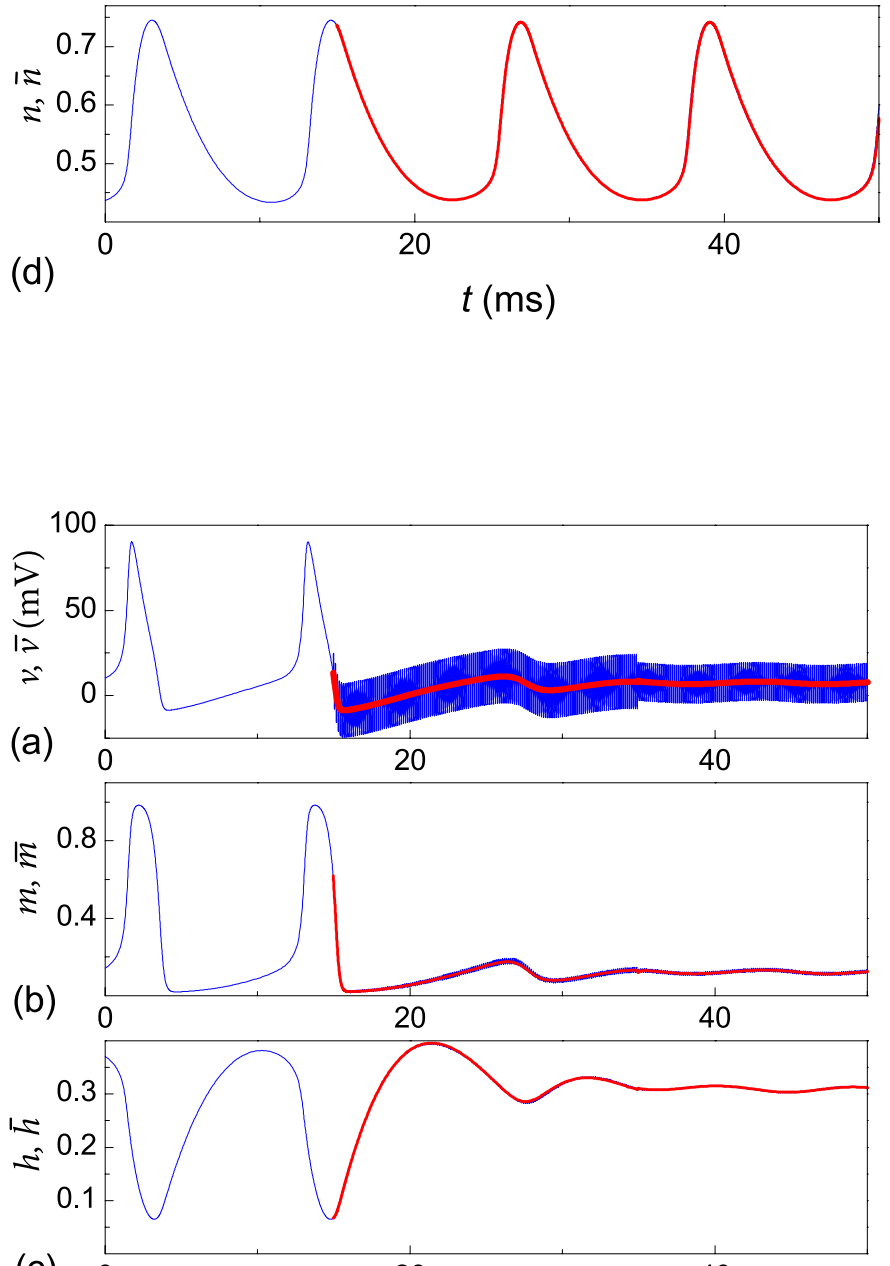

(c) \begin{tabular}{lll}
1 & 1 \\
\hline & 20 & 40
\end{tabular}

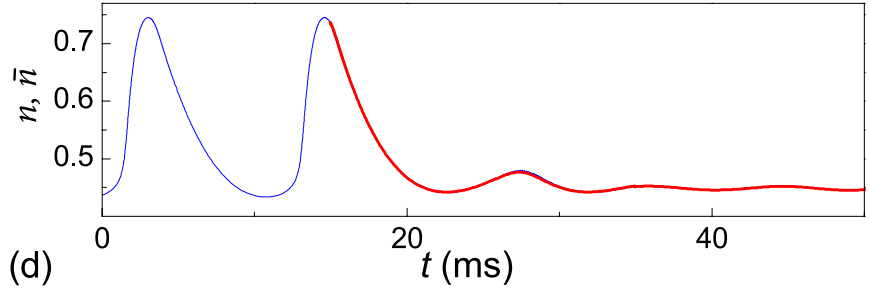

Fig. 7. The influence of HFS of moderate intensity $A_{\text {subH }}<A<A_{\text {dc }}$ on the Hodgkin-Huxley neuron dynamics. The HFS of amplitude $a=560 \mathrm{~mA} / \mathrm{cm}^{2}$ and frequency $\omega=50 \mathrm{kHz}(A=11.2 \mathrm{mV})$ are switched on at $t=t_{\mathrm{s}}=15 \mathrm{~ms}$. Thin and bold lines show the solutions of Eqs. (20) and (23), respectively.

Fig. 8. Suppression of spontaneous firing in the Hodgkin-Huxley neuron with HFS of moderate intensity via a two-step algorithm. For $t<t_{s}=15 \mathrm{~ms}$ the free system $(a=0)$ generates spontaneous spikes corresponding to the stable limit cycle shown in Fig. 6(a). At $t=t_{s}=15 \mathrm{~ms}$ the HFS of a large amplitude $a=800 \mathrm{~mA} / \mathrm{cm}^{2}\left(A=16 \mathrm{mV}>A_{d c}\right)$ is switched on. At $t=35 \mathrm{~ms}$ the amplitude is decreased to the value $a=560 \mathrm{~mA} / \mathrm{cm}^{2}$, the same as in Fig. 7 . 
gumo and Morris-Lecar neurons as well as the fourdimensional Hodgkin-Huxley model. The generic nature of this effect is further substantiated by our previous findings in isolated subthalamic nucleus model neurons [27]. This phenomenon is common not only for neuronal systems, but it is typical for any self-sustained oscillator. The analysis of the normal form of the supercritical Hopf bifurcation extended by a high-frequency excitation term shows that the bifurcation point can effectively be controlled by the excitation intensity.

To analyze the neuron equations in the presence of high-frequency stimulation we have applied a multiple-scale method. We have shown that the solution consists of a sum of slow and fast components. Similar separations of fast and slow motions is typical for many problems considered in vibrational mechanics. In our case the fast "vibrational" component has a significant amplitude only for the membrane potential while for the recovery variables it is small. The slow component changes only slightly in one period of stimulation and describes the system dynamics averaged over the period of the fast stimulation. We have derived the averaged equations governing the slow dynamics of the system for a general neuron model.

By means of analyses of the bifurcations in the averaged equations we have explained the mechanism of inhibition/blockage of the neuronal spontaneous activity. The inhibition/blockage occurs due to a stabilization of the resting state of the slow motion. With an increase of intensity of high-frequency stimulation the resting state can be stabilized by different scenarios, depending on the specific model. For the FitzHughNagumo and Morris-Lecar models the stabilization appears through a reverse supercritical Hopf bifurcation. For the Hodgkin-Huxley neuron a more complicated scenario was found. The system undergoes two successive bifurcations, the reverse subcritical Hopf bifurcation and the double-cycle bifurcation. Between these two bifurcations the bistable regime prevails where the neuron may generate stable periodic spikes or be in a stable resting state.

The averaged neuron equations derived in this paper are useful not only for understanding the effects of the high-frequency stimulation, but they are also important for the simplification of the numerical analysis. The solution of the original neuron equations with the high-frequency term varies on two different time-scales. An accurate numerical analysis of such systems requires special numerical methods and large computation times. In contrast, the averaged equations contain only the slow time-scale and their numerical simulation is simple. The averaged equations may be particularly convenient for problems where a numerical analysis of large neuronal networks under the action of high-frequency stimulation is required.

In this paper, we have restricted ourselves to the analysis of the influence of high-frequency stimulation on the single neuron models. Such models are obviously incomplete which might hinder a thorough interpretation of experimental results in the context of high-frequency deep brain stimulation of parkinsonian patients. Nevertheless, already this comparably simple one-neuron model reproduces relevant experimental findings. For instance, it was shown that electrical deep brain stimulation at frequencies greater than $100 \mathrm{~Hz}$ effectively suppresses essential tremor and parkinsonian tremor, whereas low-frequency deep brain stimulation (at frequencies below $50 \mathrm{~Hz}$ ) does not induce a tremor suppression and may even enhance the tremor when delivered at frequencies around $5-10 \mathrm{~Hz}[6,33,34]$. In a next step, our approach should be applied to the analysis of neuronal networks subjected to highfrequency stimulation in order to complement numerical simulations [35, 36] and provide a thorough understanding of the differential effects of high-frequency stimulation on physiologically relevant slow and fast neuronal dynamics.

As shown in detail above, high-frequency stimulation suppresses low-frequency oscillations, but causes relevant high-frequency oscillations of the membrane potential or corresponding variables (see Figs. 1, 3, and 5).

In Parkinson's disease synchronized neuronal oscillations emerge in different frequency bands in the basal ganglia. Synchronized local field potential (LFP) oscillations in the beta band $(13-35 \mathrm{~Hz})$ are a hallmark of Parkinson's disease [37]. A medication (levodopa) induced decrease of beta band LFP oscillations positively correlates with an improvement of akinesia and rigidity, but not with a reduction of tremor [37, 38].

Parkinsonian tremor appears to be generated by oscillatory neuronal activity in the theta band (3-7 Hz) in segregated networks associated with different limbs shaking with time-varying phase and frequency differences [39-44]. Tremor-related theta band oscillations are typically no pronounced feature in LFP recordings, presumably since different neuronal subpopulations belonging to different limbs oscillate with time varying phase and frequency relationships [41, 43, 44]. Nevertheless, with nonlinear data analysis tools it was shown that theta band (3-7 Hz) LFP oscillations drive the peripheral tremor [45]. 
Apart from the pathological low-frequency oscillations in the theta and beta range, in the basal ganglia of Parkinson's patients there are also LFP oscillations at higher frequencies. LFP oscillations in the gamma band $(35-90 \mathrm{~Hz})$ appear to contribute to the generation of (physiological) movements [37, 46]. In fact, gamma band LFP oscillations increase during movement and following appropriate (dopaminergic) medication [47-50].

In addition, the so-called high-frequency oscillations (HFO, $>200 \mathrm{~Hz}$ ) were observed in the basal ganglia of patients with Parkinson's disease [51-53]. HFO are involved in the generation of voluntary movements. The HFO power increases when movements are initiated and decreases when the latter end [51, 54. Accordingly, HFO power is negatively correlated with the akinesia/rigidity of the corresponding (contralateral) side [55].

In simple terms, the low-frequency (theta and beta) oscillations contribute to pathological processes, whereas the high-frequency oscillations (gamma and HFO) are relevant for voluntary, physiological movements. However, the low-frequency and the high-frequency oscillations interact under both physiological [56] and pathological [57] conditions. For instance, physiological processes in motor circuits may be perturbed by a coupling of the HFO amplitude [57, 58] to the phase of beta oscillations.

Based on the results obtained in our simple model presented here and in our previous study [27] we may expect high-frequency stimulation to effectively suppress low-frequency oscillations, such as theta and beta band LFP oscillations, and to induce high-frequency oscillations (in the range of the stimulation frequency) of the membrane potential. The functional role of these high-frequency, stimulus-induced oscillations in a neuronal network still remains to be investigated. However, in the context of DBS our results suggest the following testable hypotheses: (i) Standard DBS frequencies are in the range of $100-150 \mathrm{~Hz}$, often around $130 \mathrm{~Hz}$. In contrast, DBS at sufficiently high frequencies may considerably attenuate the amplitude of gamma oscillations and, hence, help to investigate the functional role of gamma. However, this requires much greater stimulation frequencies compared to the standard. (ii) Currently, studies are performed to understand fundamental, attenuating effects of high-frequency deep brain stimulation on beta band LFP oscillations [59, 60]. Nevertheless, the effects of DBS on oscillations of higher frequencies may be significant as well. DBS at standard frequencies $(100-150 \mathrm{~Hz})$ may cause linear/non-linear resonance effects and, thus, enhance neuronal oscillations at higher fre- quencies not related to the physiological context, e. g. to the onset and offset of voluntary movements. Such resonance-like effects may be detrimental. Accordingly, it is desirable to reduce the overall stimulation current. One approach aiming at this goal is to perform closed-loop, demand controlled DBS. This is to stimulate only when necessary and/or to adapt the strength of stimulation to the amount of abnormal neuronal synchrony. Initially introduced in computational studies with different types of specifically designed desynchronizing stimuli [61-63], demand-controlled DBS was experimentally tested by means of conventional high-frequency stimulation and denoted as adaptive DBS (aDBS) [64-67]. An alternative approach to significantly reduce stimulation current originated from computational studies on desynchronizing stimulation techniques $61-$ 63. 68 by incorporating spike timing-dependent plasticity (STDP) [69, 70]. Computationally it was shown that in neuronal networks with STDP coordinated reset (CR) stimulation [63], a desynchronizing stimulation technique reduces the rate of coincidences and, hence, down-regulates abnormal synaptic weights [71, 72]. CR stimulation moves the neuronal population from a pathological attractor (with strong synaptic connectivity and neuronal synchrony) to a more physiological attractor (characterized by reduced synaptic connectivity and neuronal synchrony), in this way inducing cumulative, long-lasting, sustained desynchronizing effects [7173. These computational predictions were verified both pre-clinically and clinically: long-lasting, sustained and cumulative therapeutic effects of CR-DBS were demonstrated in parkinsonian monkeys [74, 75. Long-lasting desynchronizing and therapeutic effects of CR-DBS were shown in patients with Parkinson's disease [76]. The goal of this approach is to induce an anti-kindling, i. e. to reshape the networks synaptic connectivity in a way that the network is no longer able to produce abnormal neuronal synchrony [71].

\section{Appendix: Derivation of averaged equations for a general neuron model}

We consider typical neuron models, such as the FitzHugh-Nagumo, Morris-Lecar, HodgkinHuxley or others under HFS. Generally they can be presented by the following set of equations:

$$
\begin{aligned}
& C \dot{v}=f(v, \mathbf{w})+a \varphi(\omega t), \\
& \dot{\mathbf{w}}=\mathbf{g}(v, \mathbf{w}) .
\end{aligned}
$$


Here $C$ is the membrane capacitance and $v$ is the membrane potential, $a \varphi(\omega t)$ is the HFS current, where $a$ is the amplitude and $\omega$ is the frequency. We consider a general case when $\varphi(\omega t)$ is any $2 \pi$ periodic function $\varphi(\omega t+2 \pi)=\varphi(\omega t)$, not necessary harmonic signal. In order to provide a charge-balanced stimulation, we require $\int_{0}^{\mathrm{T}} \varphi(\omega t) \mathrm{d} t=0$, where $T=2 \pi / \omega$ is the period of HFS. Equation (A.1b) describes the dynamics of a recovery variable $w$ that generally is a vector variable, $\mathbf{w}=\left(w_{1}, \ldots, w_{n}\right)$. The length $n$ of this vector as well as the functions $f$ and $\mathbf{g}$ are defined by a specific neuron model.

The derivation of averaged equations for this general case is similar to that described in Section 2. We suppose that $\omega^{-1}$ defines the shortest time-scale of the system and introduce the fast time $\tau=\omega t$. Assuming that the amplitude $a$ is proportional to $\omega$, $a=\omega C A$, we expand the solution in powers of small parameter $\omega^{-1}$ :

$$
\begin{aligned}
& v(t)=v_{0}(t, \tau)+\omega^{-1} v_{1}(t, \tau) \ldots, \\
& \mathbf{w}(t)=\mathbf{w}_{0}(t, \tau)+\omega^{-1} \mathbf{w}_{1}(t, \tau) \ldots
\end{aligned}
$$

Here functions $v_{0}(t, \tau), v_{1}(t, \tau), \mathbf{w}_{0}(t, \tau)$ and $\mathbf{w}_{0}(t, \tau)$ are $2 \pi$ periodic in $\tau$. The time-derivatives of $v$ and $\mathbf{w}$ variables are

$$
\begin{aligned}
& \dot{v}=\omega \frac{\partial v_{0}}{\partial \tau}+\frac{\partial v_{0}}{\partial t}+\frac{\partial v_{1}}{\partial \tau} \cdots \\
& \dot{\mathbf{w}}=\omega \frac{\partial \mathbf{w}_{0}}{\partial \tau}+\frac{\partial \mathbf{w}_{0}}{\partial t}+\frac{\partial \mathbf{w}_{1}}{\partial \tau} \cdots
\end{aligned}
$$

Substituting (A.2) and (A.3) into (A.1) and equating terms proportional to $\omega^{1}$ we obtain

$$
\begin{aligned}
& \frac{\partial v_{0}}{\partial \tau}=A \varphi(\tau), \\
& \frac{\partial \mathbf{w}_{0}}{\partial \tau}=0
\end{aligned}
$$

Similarly for terms proportional to $\omega^{0}$ we get

$$
\begin{aligned}
& \frac{\partial v_{0}}{\partial t}+\frac{\partial v_{1}}{\partial \tau}=C^{-1} f\left(v_{0}, \mathbf{w}_{0}\right), \\
& \frac{\partial \mathbf{w}_{0}}{\partial t}+\frac{\partial \mathbf{w}_{1}}{\partial \tau}=\mathbf{g}\left(v_{0}, \mathbf{w}_{0}\right) .
\end{aligned}
$$

The solution of Eqs. (A.4) is

$$
v_{0}(t, \tau)=\bar{v}(t)+A \psi(\tau)
$$

$$
\mathbf{w}_{0}(t, \tau)=\overline{\mathbf{w}}(t)
$$

where $\bar{v}(t)$ and $\overline{\mathbf{w}}(t)$ are any functions of the slow time $t$ and $\psi(\tau)=\int \varphi(\tau) \mathrm{d} \tau$. The equations for these functions are obtained by substituting (A.6) into Eqs. (A.5)

$$
\begin{aligned}
& \frac{\mathrm{d} \bar{v}}{\mathrm{~d} t}+\frac{\partial v_{1}}{\partial \tau}=C^{-1} f(\bar{v}+A \psi(\tau), \overline{\mathbf{w}}), \\
& \frac{\mathrm{d} \overline{\mathbf{w}}}{\mathrm{d} t}+\frac{\partial \overline{\mathbf{w}}_{1}}{\partial \tau}=\mathbf{g}(\bar{v}+A \psi(\tau), \overline{\mathbf{w}}),
\end{aligned}
$$

and averaging them over the fast time $\tau$ :

$$
\begin{aligned}
& C \dot{\bar{v}}=\langle f(\bar{v}+A \psi(\tau), \overline{\mathbf{w}})\rangle_{\tau}, \\
& \dot{\overline{\mathbf{w}}}=\langle\mathbf{g}(-v+A \psi(\tau), \overline{\mathbf{w}})\rangle_{\tau} .
\end{aligned}
$$

Here the angle brackets denote the averaging over the period of the fast time, $\langle(\cdots)\rangle_{\tau}=(1 / 2 \pi) \int_{0}^{2 \pi}(\cdots) \mathrm{d} \tau$. Finally, an approximate solution of Eqs. (A.1) is

$$
\begin{aligned}
& v(t) \approx \bar{v}(t)+A \psi(\omega t), \\
& \mathbf{w}(t) \approx \overline{\mathbf{w}}(t),
\end{aligned}
$$

where $A=a / \omega C$ and the functions $\bar{v}(t)$ and $\overline{\mathbf{w}}(t)$ satisfy the averaged equations (A.8). If the HFS is performed by the harmonic signal $\varphi(\omega t)=\cos (\omega t)$, then $\psi(\tau)=\sin (\tau)$.

\section{References}

[1] A. Stephenson, On a new type of dynamical stability, Mem. Proc. Manch. Lit. Phil. Soc. 52, 1-10 (1908).

[2] A. Stephenson, On induced stability, Phil. Mag. 15, 233-236 (1908).

[3] P.L. Kapitsa, Dynamic stability of a pendulum when its point of suspension vibrates, Sov. Phys. JETP 21, 588-592 (1951).

[4] P.L. Kapitsa, Pendulum with a vibrating suspension, Usp. Fiz. Nauk 44, 7-15 (1951).

[5] Selected Topics in Vibrational Mechanics, ed. I. Blekhman (World Scientific, New Jersey, 2003).

[6] A.L. Benabid, P. Pollak, C. Gervason, D. Hoffmann, D.M. Gao, M. Hommel, J.E. Perret, and J. de Rougemont, Long-term suppression of tremor by chronic stimulation of ventral intermediate thalamic nucleus, Lancet 337, 403-406 (1991).

[7] G. Deuschl, C. Schade-Brittinger, P. Krack, J. Volkmann, H. Schäfer, K. Bötzel, C. Daniels, A. Deutschländer, U. Dillmann, W. Eisner, et al., 
A randomized trial of deep-brain stimulation for Parkinson's disease, N. Engl. J. Med. 1355, 896908 (2006).

[8] P. Krack, A. Batir, N. Van Blercom, S. Chabardes, V. Fraix, C. Ardouin, A. Koudsie, P.D. Limousin, A. Benazzouz, J.F. LeBas, A.L. Benabid, and P. Pollak, Five-year follow-up of bilateral stimulation of the subthalamic nucleus in advanced Parkinson's disease, N. Engl. J. Med. 349, 1925-1934 (2003).

[9] P. Limusin, P. Krack, and P. Pollak, A. Benazzouz, C. Ardouin, D. Hoffmann, and A.-L. Benabid, Electrical stimulation of the subthalamic nucleus in advanced Parkinson's disease, N. Engl. J. Med. 339, 1105-1111 (1998).

[10] P. Limousin, P. Pollak, A. Benazzouz, D. Hoffmann, J.F. Le Bas, E. Broussolle, J.E. Perret, and A.L. Benabid, Effect of parkinsonian signs and symptoms of bilateral subthalamic nucleus stimulation, Lancet 345, 91-95 (1995).

[11] M.C. Rodriguez-Oroz, A. Gorospe, J. Guridi, E. Ramos, G. Linazasoro, M. Rodriguez-Palmero, and J.A. Obeso, Bilateral deep brain stimulation of the subthalamic nucleus in Parkinson's disease, Neurology 55(12 Suppl 6), S45-51 (2000).

[12] A.M. Lozano, J. Dostrovsky, R. Chen, and P. Ashby, Deep brain stimulation for Parkinson's disease: disrupting the disruption, Lancet Neurol. 1, 225231 (2002).

[13] W.J. Marks, Deep brain stimulation for dystonia, Curr. Treat. Options Neurol. 7, 237-243 (2005).

[14]C. Hamani, J.M. Schwalb, A.R. Rezai, J.O. Dostrovsky, K.D. Davis, and A.M. Lozano, Deep brain stimulation for chronic neuropathic pain: Longterm outcome and the incidence of insertional effect, Pain 125, 188-196 (2006).

[15] J.L. Viek, Mechanism of deep brain stimulation: excitation or inhibition, Mov. Disord. 17(3), S6972 (2002).

[16] A.L. Benabid, A. Benazzous, and P. Pollak, Mechanisms of deep brain stimulation, Mov. Disord. 17(3), S73-74 (2002).

[17] A.M. Lozano and H. Eltahawy, How does DBS work? Suppl. Clin. Neurophysiol. 57, 733-736 (2004).

[18] C. Beurrier, B. Bioulac, J. Audin, and C. Hammond, High-frequency stimulation produces a transient blockade of voltage-gated currents in subthalamic neurons, J. Neurophysiol. 85, 1351-1356 (2001).

[19] C. Magarinos-Ascone, J.H. Pazo, O. Macadar, and W. Buno, High-frequency stimulation of the subthalamic nucleus silences subthalamic neurons: a possible cellular mechanism in Parkinson's disease, Neuroscience 115, 1109-1117 (2002).

[20] A. Benazzouz, D.M. Gao, Z.G. Ni, B. Piallat, R. Bouali-Benazzouz, and A.L. Benabid, Effect of high-frequency stimulation of the subthalamic nucleus on the neuronal activities of the substantia nigra pars reticulata and ventrolateral nucleus of the thalamus in the rat, Neuroscience 99, 289-295 (2000).

[21]T. Boraud, E. Bezard, B. Bioulac, and C. Gross, High frequency stimulation of the internal Globus Pallidus (GPi) simultaneously improves parkinsonian symptoms and reduces the firing frequency of GPi neurons in the MPTP-treated monkey, Neurosci. Lett. 215, 17-20 (1996).

[22] C.H. Tai, T. Boraud, E. Bezard, B. Bioulac, C. Gross, and A. Benazzouz, Electrophysiological and metabolic evidence that high-frequency stimulation of the subthalamic nucleus bridles neuronal activity in the subthalamic nucleus and the substantia nigra reticulata, FASEB J. 17, 1820-1830 (2003).

[23]J.O. Dostrovsky, R. Levy, J.P. Wu, W.D. Hutchison, R.R. Tasker, and A.M. Lozano, Microstimulationinduced inhibition of neuronal firing in human globus pallidus, J. Neurophysiol. 84, 570-574 (2000).

[24] M. Filali, W.D. Hutchison, V.N. Palter, A.M. Lozano, and J.O. Dostrovsky, Stimulation-induced inhibition of neuronal firing in human subthalamic nucleus, Exp. Brain. Res. 156, 274-281 (2004).

[25]R. Levy, A.E. Lang, J.O. Dostrovsky, P. Pahapill, J. Romas, J. Saint-Cyr, W.D. Hutchison, and A.M. Lozano, Lidocaine and muscimol microinjections in subthalamic nucleus reverse Parkinsonian symptoms, Brain 124, 2105-2118 (2001).

[26]M.L. Welter, J.L. Houeto, A.M. Bonnet, P.B. Bejjani, V. Mesnage, D. Dormont, S. Navarro, P. Cornu, Y. Agid, and B. Pidoux, Effects of high-frequency stimulation on subthalamic neuronal activity in parkinsonian patients, Arch. Neurol. 61, 89-96 (2004).

[27]K. Pyragas, V. Novičenko, and P.A. Tass, Mechanism of suppression of sustained neuronal spiking under high-frequency stimulation, Biol. Cybern. 107, 669-684 (2013).

[28] R. FitzHugh, Impulses and physiological states in theoretical models of nerve membrane, Biophys. J. 1, 445-466 (1961).

[29] C. Morris and H. Lecar, Voltage oscillations in the barnacle giant muscle fiber, Biophys. J. 35, 193-213 (1981).

[30] A.L. Hodgkin and A.F. Huxley, A quantitative description of membrane current and its application to conduction and excitation in nerve, J. Physiol. 117, 500-544 (1952).

[31]J. Kevorkian and J.D. Cole, Multiple Scale and Singular Perturbation Methods (Springer-Verlag, New York, 1996).

[32] W. Gerstner and W.M. Kistler, Spiking Neuron Models. Single Neurons, Populations, Plasticity (Cambridge University Press, 2002).

[33] A.L. Benabid, P. Pollak, A. Louveau, S. Henry, and J. de Rougemont, Combined (thalamotomy and stimulation) stereotactic surgery of the VIM thalamic nucleus for bilateral Parkinson disease, Appl. Neurophysiol. 50, 344-346 (1987). 
[34]A.L. Benabid, Deep brain stimulation for Parkinson's disease, Curr. Opin. Neurobiol. 13, 696-706 (2003).

[35]D. Terman, J.E. Rubin, A.C. Yew, and C.J. Wilson, Activity patterns in a model for the subthalamopallidal network of the basal ganglia, J. Neurosci. 22, 2963-2976 (2002).

[36]C. Hauptmann and P.A. Tass, Therapeutic rewiring by means of desynchronizing brain stimulation, Biosystems 89, 173-181 (2007).

[37] P. Brown and D. Williams, Basal ganglia local field potential activity: character and functional significance, Clin. Neurophysiol. 116, 2510-2519 (2005).

[38] A.A. Kühn, A. Kupsch, G.H. Schneider, and P. Brown, Reduction in subthalamic $8-35 \mathrm{~Hz}$ oscillatory activity correlates with clinical improvement in Parkinson's disease, Eur. J. Neurosci. 23, 1956-1960 (2006).

[39] W.W. Alberts, E.W. Wright Jr, and B. Feinstein, Cortical potentials and Parkinsonian tremor, Nature 221, 670-672 (1969).

[40] H. Ben-Pazi, H. Bergman, J.A. Goldberg, N. Giladi, D. Hansel, A. Reches, and E.S. Simon, Synchrony of rest tremor in multiple limbs in Parkinson's disease: evidence for multiple oscillators, J. Neural Transm. 108, 287-296 (2001).

[41]J.M. Hurtado, C.M. Gray, L.B. Tamas, and K.A. Sigvardt, Dynamics of tremor-related oscillations in the human globus pallidus: a single case study, Proc. Natl. Acad. Sci. USA 96, 1674-1679 (1999).

[42] J.M. Hurtado, J.P. Lachaux, D.J. Beckley, C.M. Gray, and K.A. Sigvardt, Inter- and intralimb oscillator coupling in parkinsonian tremor, Mov. Disord. 15, 683-691 (2000).

[43]J.M. Hurtado, L.L. Rubchinsky, K.A. Sigvardt, V.L. Wheelock, and C.T. Pappas, Temporal evolution of oscillations and synchrony in GPi/muscle pairs in Parkinson's disease, J. Neurophysiol. 93, 1569-1584 (2005).

[44] R. Levy, W.D. Hutchison, A.M. Lozano, and J.O. Dostrovsky, High-frequency synchronization of neuronal activity in the subthalamic nucleus of Parkinsonian patients with limb tremor, J. Neurosci. 20, 7766-7775 (2000).

[45] P.A. Tass, D. Smirnov, A. Karavaev, U. Barnikol, T. Barnikol, I. Adamchic, C. Hauptmann, N. Pawelcyzk, M. Maarouf, V. Sturm, H.J. Freund, and B. Bezruchko, The causal relationship between subcortical local field potential oscillations and Parkinsonian resting tremor, J. Neural Eng. 7, 016009 (2010).

[46] P. Brown, Oscillatory nature of human basal ganglia activity: relationship to the pathophysiology of Parkinson's disease, Mov. Disord. 18, 357-363 (2003).

[47]F. Alonso-Frech, I. Zamarbide, M. Alegre, M.C. Rodríguez-Oroz, J. Guridi, M. Manrique,
M. Valencia, J. Artieda, and J.A. Obeso, Slow oscillatory activity and levodopa-induced dyskinesias in Parkinson's disease, Brain 129, 1748-1757 (2006).

[48] A.G. Androulidakis, A.A. Kuhn, C.C. Chen, P. Blomstedt, F. Kempf, A. Kupsch, G.H. Schneider, L. Doyle, P. Dowsey-Limousin, M.I. Hariz, and P. Brown, Dopaminergic therapy promotes lateralized motor activity in the subthalamic area in Parkinson's disease, Brain 130, 457-468 (2007).

[49] M. Cassidy, P. Mazzone, A. Oliviero, A. Insola, P. Tonali, V. Di Lazzaro, and P. Brown, Movementrelated changes in synchronization in the human basal ganglia, Brain 125, 1235-1246 (2002).

[50] D. Williams, M. Tijssen, G. Van Bruggen, A. Bosch, A. Insola, V. Di Lazzaro, P. Mazzone, A. Oliviero, A. Quartarone, H. Speelman, and P. Brown, Dopamine-dependent changes in the functional connectivity between basal ganglia and cerebral cortex in humans, Brain 125, 1558-1569 (2002).

[51] G. Foffani, G. Ardolini, B. Meda, M. Egidi, P. Rampini, E. Caputo, G. Baselli, and A. Priori, Altered subthalamo-pallidal synchronisation in parkinsonian dyskinesias, J. Neurol. Neurosurg. Psych. 76, 426-428 (2005).

[52]J. Lopez-Azcarate, M. Tainta, M.C. RodriguezOroz, M. Valencia, R. González, J. Guridi, J. Iriarte, J.A. Obeso, J. Artieda, and M. Alegre, Coupling between beta and high-frequency activity in the human subthalamic nucleus may be a pathophysiological mechanism in Parkinson's disease, J. Neurosci. 30, 6667-6677 (2010).

[53]T.E. Özkurt, M. Butz, M. Homburger, S. Elben, J. Vesper, L. Wojtecki, and A. Schnitzler, High frequency oscillations in the subthalamic nucleus: a neurophysiological marker of the motor state in Parkinson's disease, Exp. Neurol. 229, 324-331 (2011).

[54] H. Tan, A. Pogosyan, A. Anzak, K. Ashkan, M. Bogdanovic, A.L. Green, T. Aziz, T. Foltynie, P. Limousin, L. Zrinzo, and P. Brown, Complementary roles of different oscillatory activities in the subthalamic nucleus in coding motor effort in Parkinsonism, Exp. Neurol. 248, 187-195 (2013).

[55]J. Wang, J. Hirschmann, S. Elben, C.J. Hartmann, J. Vesper, L. Wojtecki, and A. Schnitzler, Highfrequency oscillations in Parkinson's disease: spatial distribution and clinical relevance, Mov. Disord. 29, 1265-1272 (2014).

[56]T. Yanagisawa, O. Yamashita, M. Hirata, H. Kishima, Y. Saitoh, T. Goto, T. Yoshimine, and Y. Kamitani, Regulation of motor representation by phase-amplitude coupling in the sensorimotor cortex, J. Neurosci. 32, 15467-15475 (2012).

[57] A.I. Yang, N. Vanegas, C. Lungu, and K.A. Zaghloul, Beta-coupled high-frequency activity and betalocked neuronal spiking in the subthalamic nucleus of Parkinson's disease, J. Neurosci. 34, 1281612827 (2014). 
[58]B.C. van Wijk, M. Beudel, A. Jha, A. Oswal, T. Foltynie, M.I. Hariz, P. Limousin, L. Zrinzo, T.Z. Aziz, A.L. Green, P. Brown, and V. Litvak, Subthalamic nucleus phase-amplitude coupling correlates with motor impairment in Parkinson's disease, Clin. Neurophysiol. 127, 2010-2019 (2016).

[59]H. Bronte-Stewart, C. Barberini, M.M. Koop, B.C. Hill, J.M. Henderson, and B. Wingeier, The STN beta-band profile in Parkinson's disease is stationary and shows prolonged attenuation after deep brain stimulation, Exp. Neurol. 215, 2028 (2009).

[60]E.J. Quinn, Z. Blumenfeld, A. Velisar, M.M. Koop, L.A. Shreve, M.H. Trager, B.C. Hill, C. Kilbane, J.M. Henderson, and H. Brontë-Stewart, Beta oscillations in freely moving Parkinson's subjects are attenuated during deep brain stimulation, Mov. Disord. 30, 1750-1758 (2015).

[61] P.A. Tass, Stochastic phase resetting: a theory for deep brain stimulation, Prog. Theor. Phys. Suppl. (Kyoto) 139, 301-313 (2000).

[62] P.A. Tass, Effective desynchronization by means of double-pulse phase resetting, Europhys. Lett. 53, 15-21 (2001).

[63] P.A. Tass, A model of desynchronizing deep brain stimulation with a demand-controlled coordinated reset of neural subpopulations, Biol. Cybern. 89, 81-88 (2003).

[64] S. Little and P. Brown, What brain signals are suitable for feedback control of deep brain stimulation in Parkinson's disease? Ann. N. Y. Acad. Sci. 1265, 9-24 (2012).

[65] S. Little, A. Pogosyan, S. Neal, B. Zavala, L. Zrinzo, M. Hariz, T. Foltynie, P. Limousin, K. Ashkan, J. FitzGerald, A.L. Green, T.Z. Aziz, and P. Brown, Adaptive deep brain stimulation in advanced Parkinson disease, Ann. Neurol. 74, 449-457 (2013).

[66] M. Rosa, M. Arlotti, G. Ardolino, F. Cogiamanian, S. Marceglia, A. Di Fonzo, F. Cortese, P.M. Rampini, and A. Priori, Adaptive deep brain stimulation in a freely moving Parkinsonian patient, Mov. Disord. 30, 1003-1005 (2015).
[67]S. Little, M. Beudel, L. Zrinzo, T. Foltynie, P. Limousin, M. Hariz, S. Neal, B. Cheeran, H. Cagnan, J. Gratwicke, T.Z. Aziz, A. Pogosyan, and P. Brown, Bilateral adaptive deep brain stimulation is effective in Parkinson's disease, J. Neurol. Neurosurg. Psychiatr. 87, 717-721 (2016).

[68] P.A. Tass, Phase Resetting in Medicine and Biology (Springer Verlag, Berlin, 1999).

[69] W. Gerstner, R. Kempter, J. van Hemmen, and $\mathrm{H}$. Wagner, A neuronal learning rule for submillisecond temporal coding, Nature 383, 76-81 (1996).

[70]H. Markram, J. Lübke, M. Frotscher, and B. Sakmann, Regulation of synaptic efficacy by coincidence of postsynaptic APs and EPSPs, Science 275, 213-215 (1997).

[71] P.A. Tass and M. Majtanik, Long-term anti-kindling effects of desynchronizing brain stimulation: a theoretical study, Biol. Cybern. 94, 58-66 (2006).

[72] P.A. Tass and C. Hauptmann, Therapeutic modulation of synaptic connectivity with desynchronizing brain stimulation, Int. J. Psychophysiol. 64, 53-61 (2007).

[73]C. Hauptmann and P.A. Tass, Cumulative and after-effects of short and weak coordinated reset stimulation - a modeling study, J. Neural Eng. 6, 016004 (2009).

[74]P.A. Tass, L. Qin, C. Hauptmann, S. Dovero, E. Bezard, T. Boraud, and W.G. Meissner, Coordinated reset neuromodulation has sustained after-effects in parkinsonian monkeys, Ann. Neurol. 72, 816-820 (2012).

[75] J. Wang, S. Nebeck, A. Muralidharan, M.D. Johnson, J.L. Vitek, and K.B. Baker, Coordinated reset deep brain stimulation of subthalamic nucleus produces long-lasting, dose-dependent motor improvements in the 1-methyl-4-phenyl-1,2,3,6tetrahydropyridine non-human primate model of parkinsonism, Brain Stimul. 9, 609-617 (2016).

[76] I. Adamchic, C. Hauptmann, U.B. Barnikol, S. Dovero, E. Bezard, T. Boraud, and W.G. Meissner, Coordinated reset has lasting aftereffects in patients with Parkinson's disease, Mov. Disord. 29, 1679-1684 (2014). 


\title{
SAVAIMINIŲ VIRPESIŲ SLOPINIMAS AUKŠTU DAŽNIU ŽADINAMUOSE NEURONUOSE
}

\author{
K. Pyragas ${ }^{a}$, P.A. Tass ${ }^{b, c, d}$ \\ ${ }^{a}$ Fiziniu ir technologijos moksly centras, Vilnius, Lietuva \\ bulicho tyrimu centras, Julichas, Vokietija \\ ' Stanfordo universitetas, Stanfordas, Kalifornija, JAV \\ ${ }^{\mathrm{d}}$ Kelno universitetas, Kelnas, Vokietija
}

\section{Santrauka}

Nagrinëjame aukšto dažnio srovès ittaką savaiminiam neuronų aktyvumui ir parodome, kad ši srovė gali nuslopinti neuronų savaiminius žemo dažnio virpesius. Šio reiškinio universalumą demonstruojame naudodami standartinius neuronu modelius (FitzHugh-Nagumo, Morris-Lecar ir Hodgkin-Huxley) ir normalinę superkrizinès Hopfo bifurkacijos formą. Taikydami skirtingų laiko mastelių metodą, mes atskiriame greitus ir lètus neuroninių lygčių sprendinius ir lètoms spren- dinių komponentėms išvedame vidurkintas lygtis. Neuroninio aktyvumo slopinimo mechanizmą aiškiname remdamiesi vidurkintų lygčių bifurkacijų analize. Gauti rezultatai padeda suprasti giluminès smegenų aukšto dažnio stimuliacijos (high-frequency deep brain stimulation) gydomaji efektą. Šiuo metu giluminė aukšto dažnio smegenų stimuliacija yra standartinè procedūra gydant Parkinsono ir kitas neurologines ligas. Mùsų tyrimai leidžia toliau tobulinti giluminès aukšto dažnio smegenų stimuliacijos metodą. 